\title{
The experimental study on the electrical characteristics of flame applied to the measurement of rate of heat release in combustion
}

\author{
Tianyu Zhang ${ }^{1,}$, Ying Feng ${ }^{2}$, Zhenning Zhao ${ }^{1}$ \\ ${ }^{1}$ North China Electric Power Research Institute, Beijing 100032, China \\ ${ }^{2}$ Institute of Economics and Technology of State Grid Chengdu power supply company, Chengdu \\ 610000 , China
}

*Corresponding author Email: zzztttyyy456@126.com

Keywords: combustion, electrical characteristics, rate of heat release

\begin{abstract}
The stability and efficiency of combustion determine utility of fuel and the rate of heat release, which is used to evaluate the performance of combustion organization. However, the walls of low temperature and fire-retardant material are prone to absorb the activated molecules, leading to break of chain reaction and incomplete combustion, which is called flame quenching. This paper mainly investigates the release of $\mathrm{CO}$, the distance of polar plates and value of current during the flame quenching process. In present study, electric field are exerted on the flame, measuring the variation of current and rate of heat release, in order to determine the optimum distance and maximum rate of heat release.
\end{abstract}

\section{Introduction}

Combustion efficiency is the ratio of actual heat release to complete heat release of unit fuel, which is a major index of evaluating the performance of combustion. Besides the characteristics of fuel, the combustion efficiency is, to great extent, determined by boundaries of combustion chamber as well. The low temperature boundaries or fire-retardant material can absorb activated molecules and lead to decrease in combustion efficiency, even flame quenching.

Meanwhile, the study of electrical characteristics of flame has drawn great attention. Some researchers pointed out that for the flame of fossil fuels, due to the existence of redundant ionization in the front and difference in diffuse velocity of positive and negative ions, external electrical field will cause the ions move faster and exert a force on the flue to fasten the reaction ratio [1]. Felix Weinberg studied the formation of synthesis gas and release of CO during the combustion process of $\mathrm{CH} 4$, and compared the results with the combustion under the electrical field [2, 3]. They drew a conclusion that the quenching process is related with the release of $\mathrm{CO}$ and current value, indicating the prediction for combustion efficiency based on the current is feasible[4, 5].

The release of CO is related with combustion efficiency and rate of heat release, based on which, present study brings out a method of diagnosis of combustion efficiency by connecting the electrical characteristics with rate of heat release. Our study takes propane as fuel, and plate materials are selected as stainless steel, the voltage of the plates varies from 9-90V.

\section{Preparation and experiment procedure}

Our experimental setup is shown in Fig.1. The propane and air are pumped respectively and join in the mixer chamber, and then to the burner. A vessel of metal is put in front of the burner and the distance between the side wall of the vessel and burner can be adjusted. A thermocouple in inserted into the vessel to obtain the combustion temperature and CO concentration measurement apparatus is placed at the end of the flue. The external electrical field is added by a set of battery, and an ammeter is put into use. 


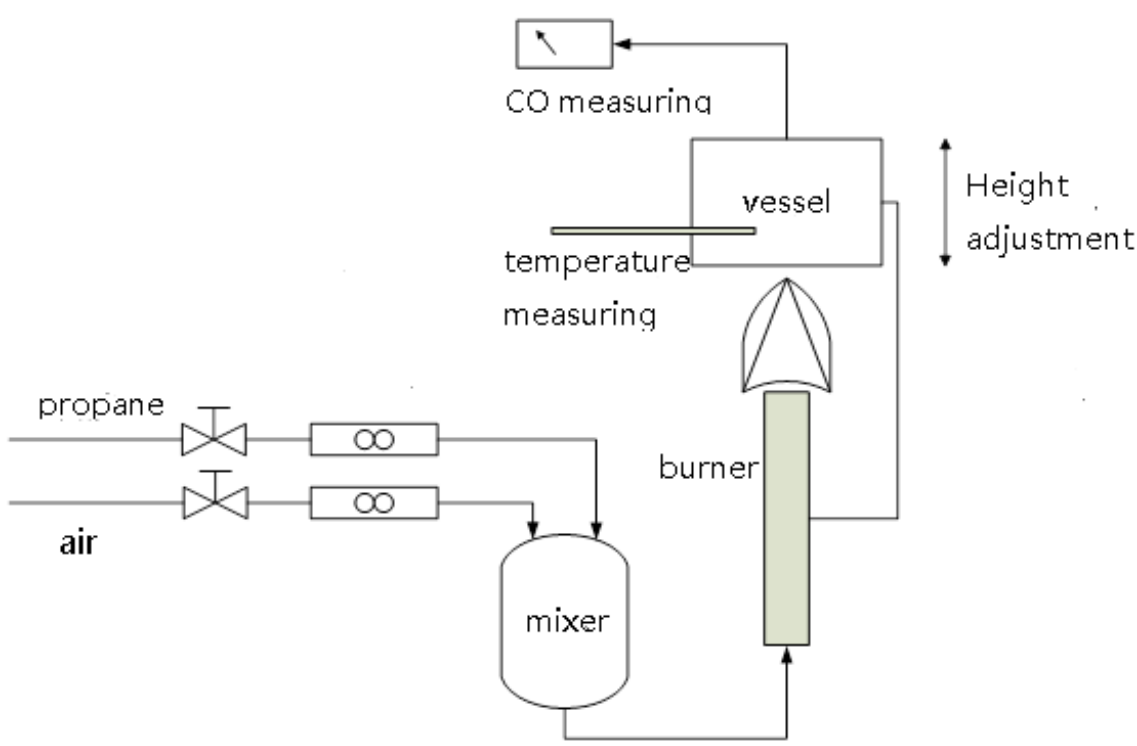

Fig.1 Diagrammatic sketches of the experiment setup

Keep the flux of propane and air constant: 1 ) investigate the relation of strength of electrical field and distance of the vessel to burner, and 2) under the electrical field, the relation between the combustion efficiency and the distance.

\section{Results and analysis}

The change of current with distance in different external battery.

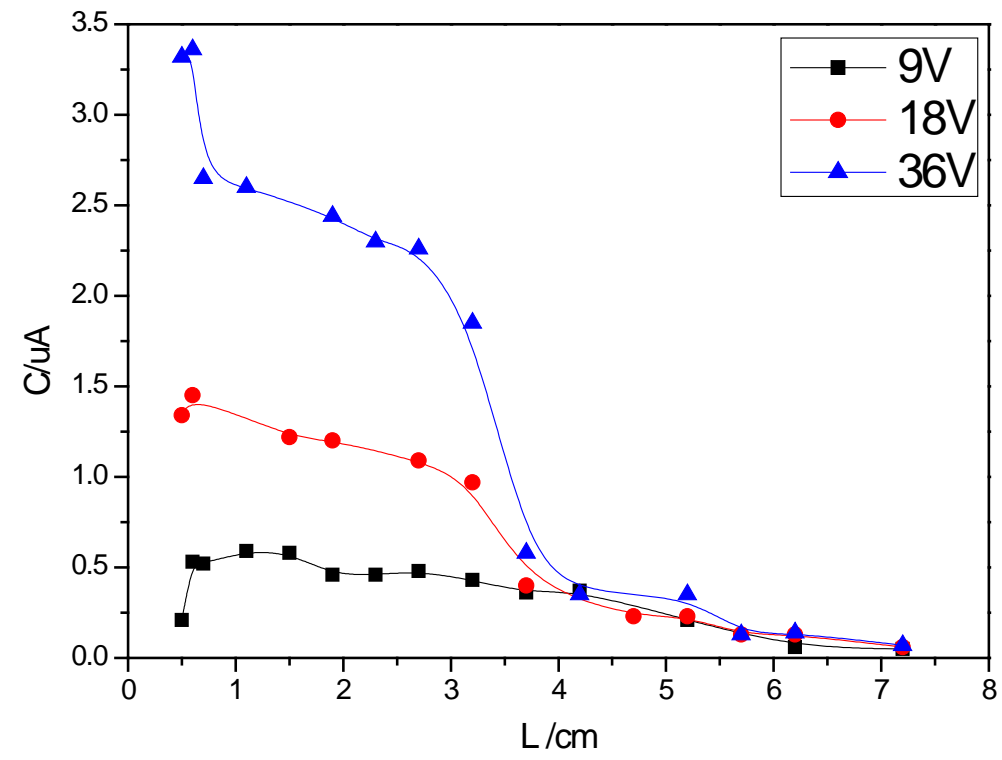

Fig.2 Change of current with distance of vessel

As shown above, the current decreases as the distance increases. When the distance is less than $4 \mathrm{~cm}$, the current is prone to reach a steady state, whose characteristics can be applied to predict the change in distance by measuring current in the ammeter. Meanwhile, we can also observe that the higher voltage is, the higher current is under same diameter. 
The time of temperature rising from $20^{\circ} \mathrm{C}$ to $50^{\circ} \mathrm{C}$ of $100 \mathrm{ml}$ water.

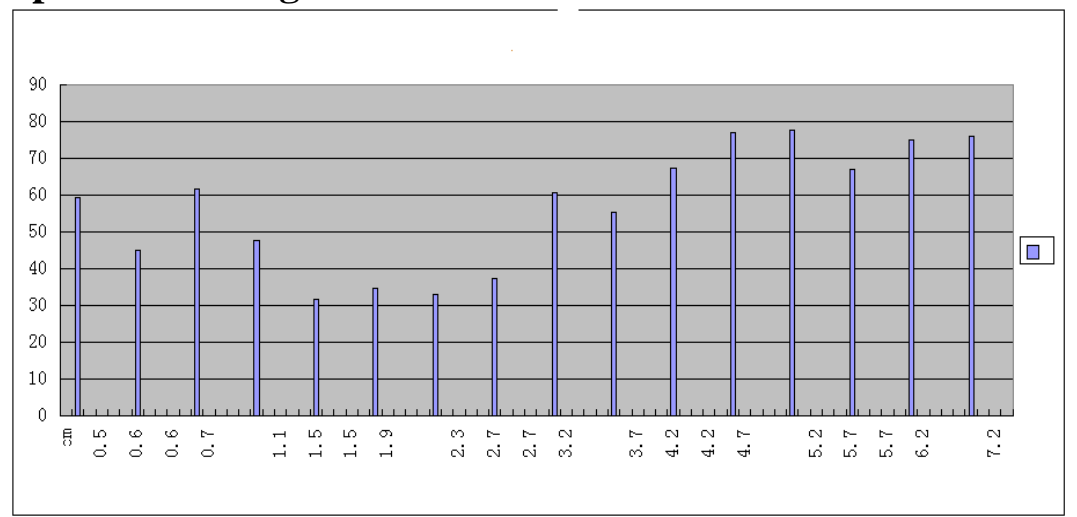

Fig.3 Time of releasing same amount of heat under different distance

As shown in Fig.2, the time of releasing same amount of heat can indicate the combustion efficiency under same fuel flux. The time first decreases and reach a bottom before increasing, which means the according combustion efficiency first increases then reach a maximum peak, and decreases.

Under the external battery of different voltage, the change of current and combustion efficiency in different distance.

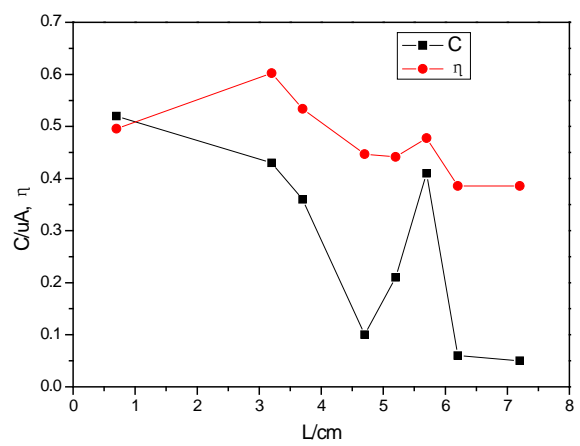

Fig.4 The change of current and combustion efficiency under different distance (9V)

Under the voltage of $9 \mathrm{~V}$, as the distance increases, except for specific point (distance from $0.5 \mathrm{~cm}$ to $3 \mathrm{~cm}$ ), the current and efficiency show nearly the same inclination. The maximum and minimum efficiency are $60 \%$ and $40 \%$, respectively. And the efficiency first increases then decreases, finally reaching a steady state. The most important point is the detection of the same inclination of efficiency and current, which can be used to reflect the instantaneous combustion efficiency by measuring the current.

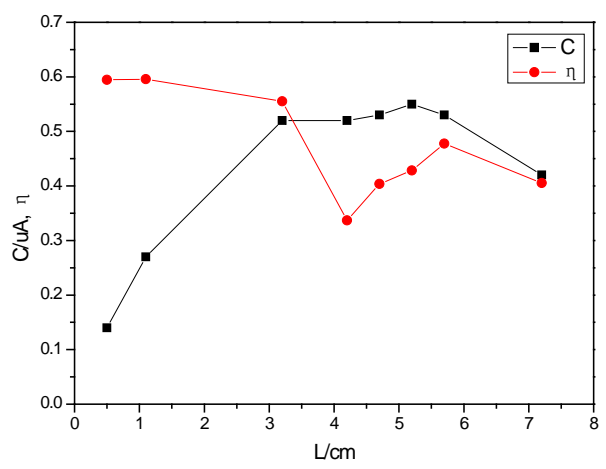

Fig.5 The change of current and combustion efficiency under different distance (18V)

As shown above, the discrepancy of inclination lies in distance between $0.5 \mathrm{~cm}$ to $2 \mathrm{~cm}$, then after $3 \mathrm{~cm}$, the current and efficiency have the same inclination. 


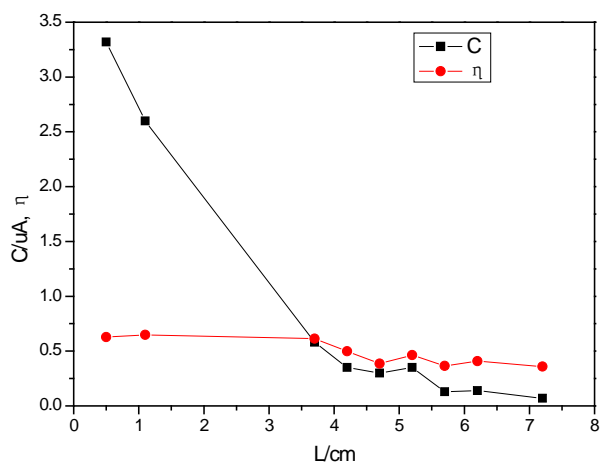

Fig.6 The change of current and combustion efficiency under different distance (36V)

The case of $36 \mathrm{~V}$ shows the similar condition with $18 \mathrm{~V}$ and $9 \mathrm{~V}$, namely when the distance is above certain value, the current and efficiency show great consistency.

\section{Conclusion}

In present study, the relation of distance, combustion efficiency and external electrical filed is studied. As distance varies, combustion efficiency first increases then decreases, finally reaching a steady value. The efficiency reaches to maximum of $60 \%$ at the range of $1.5-2.7 \mathrm{~cm}$. And the combustion efficiency is proved to be related with current under different external electrical field (9V, $18 \mathrm{~V}$ and $36 \mathrm{~V}$ ), which basically shows same variation inclination: after certain distance, the efficiency and current shows same change with distance.

\section{References}

[1] J.Lawton, F.J.Weinberg. Electrical Aspects of Combustion. Clarendon Press, Oxford, 1969.

[2] Felix Weinberg, Fred Carleton. Ionization and chemiluminescence during the progressive aeration of methane flames. Combustion and Flame, 156 (2009):2276-2284.

[3] Felix Weinberg, Fred Carleton, Raphael Houdmont, Derek Dunn-Rankin, Sunny Karnani. Syngas formation in methane flames and carbon monoxide release during quenching. Combustion and Flame, 158 (2011): 273-280

[4] J.F. Grcar, M.S. Day, J.B. Bell, Combust. Theor. Model. 10 (4) (2006) 559-579.

[5] M.C. Weikl, T. Seeger, R. Hierold, A. Leipertz, J. Raman Spectrosc. 38 (2007)983-988. 